\title{
Fusobacterium mortiferum
}

National Cancer Institute

\section{Source}

National Cancer Institute. Fusobacterium mortiferum. NCI Thesaurus. Code C86399.

A species of anaerobic, Gram-negative, pleomorphic rod shaped bacteria in the phylum Fusobacteria. This species is non-spore forming, indole and superoxide dismutase negative, hydrolyzes esculin, reduces nitrate, does not produce lysine decarboxylase, and is the only member of the genus to have equimolar ratios of mesolanthionine and diaminopimelic acids within the cell membrane. F. mortiferum is commensal to the human intestinal tract, but can be pathogenic in other locations. 